\title{
P-wave picking for earthquake early warning: refinement of a T[pd] method
}

\author{
$\operatorname{AUTHOR(S):~}$
}

Yamada, Masumi; Mori, Jim

\section{CITATION:}

Yamada, Masumi ... [et al]. P-wave picking for earthquake early warning: refinement of a T[pd] method. Geophysical Journal International 2022, 228(1): 387-395

\section{ISSUE DATE:}

2022-01

URL:

http://hdl.handle.net/2433/267453

\section{RIGHT:}

This article has been accepted for publication in Geophysical Journal International @: 2021 The Author(s) Published by Oxford University Press on behalf of the Royal Astronomical Society. All rights reserved. 


\title{
$P$-wave picking for earthquake early warning: refinement of a $T^{p d}$ method
}

\author{
Masumi Yamada ${ }^{\odot}$ and Jim Mori \\ Disaster Prevention Research Institute, Kyoto University, Uji, Gokasho 611-0011, Japan.E-mail: masumi@eqh.dpri.kyoto-u.ac.jp
}

Accepted 2021 August 24. Received 2021 July 13; in original form 2021 January 27

\begin{abstract}
S UMMAR Y
Detecting $P$-wave onsets for online processing is an important component for real-time seismology. As earthquake early warning systems around the world come into operation, the importance of reliable $P$-wave detection has increased, since the accuracy of the earthquake information depends primarily on the quality of the detection. In addition to the accuracy of arrival time determination, the robustness in the presence of noise and the speed of detection are important factors in the methods used for the earthquake early warning. In this paper, we tried to improve the $P$-wave detection method designed for real-time processing of continuous waveforms. We used the new $T^{p d}$ method, and proposed a refinement algorithm to determine the $P$-wave arrival time. Applying the refinement process substantially decreases the errors of the $P$-wave arrival time. Using 606 strong motion records of the 2011 Tohoku earthquake sequence to test the refinement methods, the median of the error was decreased from 0.15 to $0.04 \mathrm{~s}$. Only three $P$-wave arrivals were missed by the best threshold. Our results show that the $T^{p d}$ method provides better accuracy for estimating the $P$-wave arrival time compared to the STA/LTA method. The $T^{p d}$ method also shows better performance in detecting the $P$-wave arrivals of the target earthquakes in the presence of noise and coda of previous earthquakes. The $T^{p d}$ method can be computed quickly, so it would be suitable for the implementation in earthquake early warning systems.
\end{abstract}

Key words: Earthquake early warning; Earthquake ground motions; Earthquake hazards.

\section{INTRODUCTION}

Detecting $P$-wave onsets in continuous seismic data streams is a fundamental component of real-time seismology. Conventionally, the STA/LTA method (ratio between short-term and long-term averages) has been widely used for the $P$-wave detection (e.g. Allen 1978). Since this method is reasonably robust and computationally simple, it has long been used as the standard technique.

Since earthquake early warning systems have been established at a number of locations around the world (Allen et al. 2009), the importance of reliable $P$-wave detection has increased. Not only the accuracy of the arrival time, but also the robustness for noisy signals and the speed of detection are essential for earthquake early warning.

There have been several more sophisticated algorithms proposed recently. Hildyard et al. (2008) and Nippress et al. (2010) proposed the damped predominant period $\left(T^{p d}\right)$ method, which uses the change of frequency between the $P$-wave arrival and background noise. Methods using higher order statics have also been explored by researchers (Saragiotis et al. 2002; Galiana-Merino et al. 2008; Nippress et al. 2010). These methods are more sensitive to the change of statistical distribution of amplitudes. This means that the methods are also more affected by noise, so it is common to combine the techniques with other approaches to refine the triggers (e.g. Nippress et al. 2010; Ross \& Ben-Zion 2014). Adding multiband filters before the STA/LTA can also improve the accuracy (Satriano et al. 2008). Furthermore, autoregressive techniques (e.g. Takanami \& Kitagawa 1988, 1991; Leonard \& Kennett 1999), wavelet methods (e.g. Anant \& Dowla 1997; Hafez et al. 2010) and a singular value decomposition method (e.g. Kurzon et al. 2014) have been proposed to improve the $P$-wave discrimination. Applications of deep learning in the phase detection have also been proceeding rapidly (e.g. Ross et al. 2018; Zhu \& Beroza 2019; Mousavi et al. 2020). Although these methods show better performance, the speed of the detection after the phase arrival can be a limiting factor, which is also important for earthquake early warning systems.

In order to improve the $P$-wave detection method in real-time processing, we focus on the $T^{p d}$ method, which is simple and easily implemented for fast computations. An earthquake early warning system may be processing thousands of components at the same time; therefore, fast computation is important. The method uses recursive computation and does not require large memory. It uses only a few samples after the $P$-wave arrivals to detect the signal arrival, which enables the quick detection of the $P$ waves. We 
propose refined algorithms for the $P$-wave arrival time and test them by using the aftershock data set of the 2011 Tohoku-oki earthquake. We evaluate the results in terms of accuracy of arrival time, speed of detection and robustness to noise.

\section{DATA}

We used seismic data recorded by Hi-net and the JMA strong motion seismic network from 2011 March 1 to April 30. We selected 18 earthquakes shown in Table 1: 17 earthquakes with maximum JMA seismic intensity $\geq 5$ upper during the period, including the $M 9.0$ main shock, and the largest foreshock (M7.3) with intensity 5 lower. The table also indicates 'immediate' events that occur closely in time and have higher noise levels because of the first event. For comparison purposes, the stations where $P$-wave arrival times were manually determined by JMA were used. We selected at most 50 of the closest stations to the epicentre for each event. We used a total of 606 seismic waveforms for the evaluations of the methods. All waveforms are up-down (UD) components with 3 min duration. The JMA seismic network uses accelerometers, whereas the Hi-net uses short-period velocity seismometers. For the analyses of $P$-wave detections, acceleration records were used, so velocity records are differentiated once in the time domain. All data are sampled at $100 \mathrm{~Hz}$

\section{$3 T^{p d}$ METHOD}

\subsection{Basic algorithm}

$T^{p d}$ is a method proposed by Hildyard et al. (2008) that uses the change of frequency due to the $P$-wave arrival. Suppose that $x_{i}$ is the input signal, then $T^{p d}$ is calculated by

$T_{i}^{p d}=2 \pi \sqrt{\frac{X_{i}}{D_{i}+D_{s}}}$,

where

$X_{i}=\alpha X_{i-1}+x_{i}^{2}$,

$D_{i}=\alpha D_{i-1}+\left(\partial x_{i} / \partial t\right)^{2}$

and $\alpha$ is a damping parameter. This parameter is defined as $\alpha=$ $\exp ^{\ln (0.1) * \mathrm{~d} t / \tau_{\mathrm{w}}}$, that is the amplitude decreases to 0.1 after $\tau_{\mathrm{w}}$ seconds with a sampling period $\mathrm{d} t . D_{\mathrm{s}}$ is a constant to stablize $T^{p d}$. An appropriate choice of $D_{s}$ will reduce the value of $T^{p d}$ when $D_{i}$ is very small, and prevent large $T^{p d}$ being recorded for low-amplitude signals even when they contain long periods. According to Hildyard et al. (2008),

$D_{\mathrm{s}}=\frac{4 \pi^{2}\left\langle x_{i}^{2}\right\rangle \tau_{\mathrm{w}}}{\tau_{m x}^{2} \mathrm{~d} t}$,

where $\left\langle x_{i}^{2}\right\rangle$ denotes the average noise level squared. $T^{p d}$ will be close to $\tau_{m x}$ if the data are close to the noise level. In our case, we used the following long-time average for the recursive computation:

$\left\langle x_{i}^{2}\right\rangle=\left\langle x_{i-1}^{2}\right\rangle+\left(1-\exp ^{\ln (0.1) * \mathrm{~d} t / 100}\right) *\left(x_{i}^{2}-\left\langle x_{i-1}^{2}\right\rangle\right)$.

By following Hildyard et al. (2008), we use $\tau_{\mathrm{w}}=4.5$ and $\tau_{m x}=$ 0.019 .

Figs 1(a) and (b) show the traces of acceleration and $T^{p d}$ in quiet and noisy environments. $T^{p d}$ increases rapidly at the wave onset, and it decays slower after that. The original method uses the value of $T^{p d}$ as a trigger condition.
If we have two earthquakes consecutively, the shape of $T^{p d}$ is likely to be two step functions. Therefore, detecting the second event by a single threshold would be difficult if the two earthquakes occur closely in time. In order to detect both events, we use the maximum change of $T^{p d}$ in $3 \mathrm{~s}$ as a trigger condition:

$\Delta T_{\max }^{p d}>c_{1}$

where $\Delta T_{\max }^{p d}=\max \left\{T_{i}^{p d}-T_{j}^{p d} \mid(i-3 / \mathrm{d} t) \leq j<i\right\}$ and $c_{1}=$ 0.015. A $P$ wave is detected if $\Delta T_{\max }^{p d}$ exceeds the threshold of $c_{1}$. The most probable threshold will be discussed later.

\subsection{Refinement of the arrival time estimate}

The trigger threshold $\left(c_{1}\right)$ should be set so that it is not triggered by noise but triggered on a signal arrival as quickly as possible. In general, $T^{p d}$ increases as a function of time at around the $P$-wave arrival (see Fig. 1b). It would be more precise if we can pick the time step when $T^{p d}$ starts increasing from the noise level. In order to refine the $P$-wave arrival time, we try to find the largest change of the wave characteristics slightly prior to the detection. We propose a three-step refinement process.

Step $1 T^{p d}$ refinement in a short time window: The first step is to find a sudden change of $T^{p d}$ in a short time window. We search the time when the change of $T^{p d}$ exceeds $0.5 \Delta T_{\max }^{p d}$ within $0.15 \mathrm{~s}$ prior to the trigger. The refined time step $j$ is defined as

$$
\begin{array}{r}
\max \left\{j \mid T_{j}^{p d}<T_{i}^{p d}-0.5 \Delta T_{\max }^{p d} \cap T_{j+1}^{p d} \geq T_{i}^{p d}-0.5 \Delta T_{\max }^{p d},\right. \\
(i-0.15 / \mathrm{d} t) \leq j<i\}
\end{array}
$$

where $i$ is the triggered time step. Note that more than 82 percent of data (497/606) can find the time step $j$ within $0.15 \mathrm{~s}$ prior to the trigger.

Step $2 T^{p d}$ refinement in a long time window: If the change of $T^{p d}$ is not large enough in this short time window, we apply the second refinement scheme with a longer time window. We search the time when the change of $T^{p d}$ exceeds $0.8 \Delta T_{\max }^{p d}$ within $3 \mathrm{~s}$ prior to the trigger. The refined time step is defined as

$$
\begin{array}{r}
\max \left\{j \mid T_{j}^{p d}<T_{i}^{p d}-0.8 \Delta T_{\max }^{p d} \cap T_{j+1}^{p d} \geq T_{i}^{p d}-0.8 \Delta T_{\max }^{p d},\right. \\
(i-3 / \mathrm{d} t) \leq j<i\} .
\end{array}
$$

Step $3 \mathbf{d} \boldsymbol{T}^{p d}$ refinement: In the third step, the refined time step is further refined by the smoothed derivative of $T^{p d}\left(\mathrm{~d} T^{p d}\right)$. The refined time step $k$ is defined as the time when $\mathrm{d} T^{p d}$ exceeds a threshold within $1 \mathrm{~s}$ prior to the time step from the second refinement scheme (j):

$\max \left\{k \mid \mathrm{d} T_{k}^{p d}<c_{2} \cap \mathrm{d} T_{k+1}^{p d} \geq c_{2},(j-1 / \mathrm{d} t) \leq k<j\right\}$,

where $\mathrm{d} T_{i}^{p d}=\left(T_{i+1}^{p d}-T_{i-2}^{p d}\right) /(3 \mathrm{~d} t)$ and $c_{2}=0.01$.

Fig. 1 shows the refinement process for an emergent $P$-wave arrival in quiet (left) and noisy (right) environments, respectively. A signal was detected since the change of $T^{p d}$ is larger than 0.015 within $3 \mathrm{~s}$ (Fig. 1b).

In a quiet environment, the change of $T^{p d}$ was very sharp, and the first refinement scheme of eq. (7) was satisfied due to the sharp change (Fig. 1c, left). The next refinement process using $\mathrm{d} T^{p d}$ found the waveform arrival only $0.05 \mathrm{~s}$ after the manual detection (Fig. 1d, left).

In a noisy environment, $T^{p d}$ has an emergent onset, and eq. (7) was not satisfied in the first refinement. The second refinement process was applied, and the onset of the increase of $T^{p d}$ was found by eq. (8) (Fig. 1c, right). The third refinement process using $\mathrm{d} T^{p d}$ was not so effective due to the unstable slope of $T^{p d}$ (Fig. 1d, right). 
Table 1. List of earthquakes used in this study.

\begin{tabular}{lcccccccccc}
\hline No. & Date & Time & Lat. & Lon. & Dep. & $M$ & SI & Ndata & Err & Name \\
\hline 1 & $03 / 09 / 11$ & $11: 45: 13$ & 38.3285 & 143.2798 & 8.3 & 7.3 & $5-$ & 30 & - & Tohoku foreshock \\
2 & $03 / 11 / 11$ & $14: 46: 18$ & 38.1035 & 142.8610 & 23.7 & 9 & 7 & 50 & - & Tohoku \\
3 & $03 / 11 / 11$ & $15: 15: 34$ & 36.1208 & 141.2525 & 42.7 & 7.6 & $6+$ & 26 & - & Tohoku (immediate) \\
4 & $03 / 11 / 11$ & $16: 29: 00$ & 39.0225 & 142.3112 & 17.0 & 6.6 & $5+$ & 10 & - & Tohoku (immediate) \\
5 & $03 / 11 / 11$ & $17: 40: 54$ & 37.4240 & 141.2732 & 29.9 & 6 & $5+$ & 10 & - & Tohoku (immediate) \\
6 & $03 / 12 / 11$ & $03: 59: 15$ & 36.9860 & 138.5978 & 8.4 & 6.7 & $6+$ & 50 & 0.1 & Nagano \\
7 & $03 / 12 / 11$ & $04: 31: 55$ & 36.9488 & 138.5725 & 0.8 & 5.9 & $6-$ & 24 & 0.6 & Nagano (immediate) \\
8 & $03 / 12 / 11$ & $05: 42: 19$ & 36.9732 & 138.5905 & 3.8 & 5.3 & $6-$ & 19 & 0.5 & Nagano (immediate) \\
9 & $03 / 15 / 11$ & $22: 31: 46$ & 35.3095 & 138.7145 & 14.3 & 6.4 & $6+$ & 27 & 0.3 & Shizuoka \\
10 & $03 / 19 / 11$ & $18: 56: 48$ & 36.7837 & 140.5715 & 5.4 & 6.1 & $5+$ & 36 & 0.3 & Ibaraki \\
11 & $03 / 23 / 11$ & $07: 12: 28$ & 37.0848 & 140.7878 & 7.6 & 6 & $5+$ & 29 & 0.2 & Fukushima-Hamadori \\
12 & $03 / 23 / 11$ & $07: 34: 56$ & 37.0982 & 140.7958 & 6.7 & 5.5 & $5+$ & 29 & 3.6 & Fukushima (immediate) \\
13 & $03 / 23 / 11$ & $18: 55: 20$ & 37.1110 & 140.7607 & 9.0 & 4.7 & $5+$ & 34 & 0.4 & Fukushima \\
14 & $04 / 01 / 11$ & $19: 49: 44$ & 40.2568 & 140.3640 & 12.4 & 5 & $5+$ & 50 & 0.1 & Akita \\
15 & $04 / 07 / 11$ & $23: 32: 43$ & 38.2042 & 141.9202 & 65.9 & 7.2 & $6+$ & 49 & - & Miyagi-oki \\
16 & $04 / 11 / 11$ & $17: 16: 12$ & 36.9457 & 140.6727 & 6.4 & 7 & $6-$ & 50 & 0.1 & Fukushima-Hamadori \\
17 & $04 / 12 / 11$ & $14: 07: 42$ & 37.0525 & 140.6435 & 15.1 & 6.4 & $6-$ & 33 & 0.3 & Fukushima-Nakadori \\
18 & $04 / 16 / 11$ & $11: 19: 31$ & 36.3408 & 139.9455 & 78.8 & 5.9 & $5+$ & 50 & 0.2 & Ibaraki \\
\hline
\end{tabular}

Notes: The indices are event number, earthquake occurrence date, time, latitude, longitude, depth, JMA magnitude (moment magnitude for Tohoku earthquake), maximum JMA seismic intensity, number of waveforms used, location error of inland earthquakes between JMA catalogue and location calculated with automatic triggers of this study, and name of the earthquake from the left. 'Immediate' stands for the immediate aftershocks (within a few hours after a large earthquake). The catalogue is obtained from the Japan unified high-resolution relocated catalogue for earthquakes.

\subsection{Detriggering and retriggering}

It is necessary to establish a detriggered condition to avoid triggering due to secondary phases (such as the $S$ wave) but not to miss the $P$ wave for the next event. We detrigger (i.e. allow triggering another $P$ phase) if $T^{p d}$ is less than 0.01 and 20 s passed after the trigger.

We can trigger again (retrigger the same $P$ phase) if the following conditions are satisfied:

(1) $5 \mathrm{~s}$ passed after the previous trigger, and

(2) $\Delta T_{\max }^{p d}>c_{1}$ and $\Delta T_{\max }^{p d}>\Delta T_{\max }^{p d}$ (previous).

The second condition indicates that the $\Delta T_{\max }^{p d}$ should be larger than that of the previous trigger. In this way, we avoid a trigger by the $S$ wave, but do not miss a $P$ wave of a closely following event, if the second event is much larger than the first one. However, any signals within $5 \mathrm{~s}$ after a trigger will be ignored. Subsequent events within $5 \mathrm{~s}$ of an initial trigger will not be detected. The pseudo-code of the refined $T^{p d}$ algorithm is shown in Fig. 2.

\section{RESULTS}

We tested 606 waveforms that contain the $P$-wave arrival of a large earthquake. Each 3 min record includes the $P$-wave arrival of the target earthquake plus possible small earthquakes. Our goal is to minimize the missed $P$-wave arrivals for the target earthquakes and to suppress false triggers by noise or smaller earthquakes. We evaluate our method by the accuracy of correct triggers, missed $P$ waves and false triggers by noise, by comparison with $P$-wave arrivals manually picked by JMA.

\subsection{Accuracy of detection of $P$-wave arrival times}

Figs 3(a) and (b) show histograms of the $P$-wave detection error of the test data set before and after the refinement. The number of the correct triggers, and the intervals of the 95 and 50 percent of the data are shown in the figure. We defined a $P$-wave detection within
$2 \mathrm{~s}$ from the JMA manually determined $P$-wave arrival as a correct trigger.

Applying the refinement process decreases the error and increases the detectability. The median of the error was decreased from 0.15 to $0.04 \mathrm{~s}$. The number of missed $P$-wave arrivals for the target earthquakes was also decreased from 11 to 3. In total, 603 out of 606 waveforms were picked correctly by the $T^{p d}$ method. Fig. 3(c) shows the difference of the $P$-wave triggers before and after the refinement. The difference is about $0.1 \mathrm{~s}$ on average, but some triggers changed the arrival times by $1-2 \mathrm{~s}$ after the refinement. In general, the refinement process improves the arrival times slightly for waveforms in the quiet environment, but it is more effective for the waveforms in the noisy environment, whose $P$-wave onsets are emergent.

Fig. 4 shows the $P$-wave detection error for each earthquake. Events that occur immediately after other earthquakes (within a few minutes to few hours) have larger errors than isolated events. These events have higher noise levels because of the coda and aftershocks from the first event. Some earthquakes have a clear trend in the errors. For example, our results for the Tohoku main shock (no. 2 ) tend to have later arrival times than the manually determined $P$-wave arrivals. This earthquake has very emergent onsets of the $P$ waves due to 'noise' caused by the source characteristics and farther epicentre distance compared to other events. An immediate aftershock of the Fukushima-Hamadori earthquake (no. 12) has earlier arrival times than the JMA arrival times. We checked the original waveforms and found there were two $P$-wave arrivals in a very short time $(<1 \mathrm{~s})$. This may be due to a small earthquake not in the JMA catalogue. It is difficult to distinguish a small event before a target earthquake, since we do not allow retriggering for $5 \mathrm{~s}$ after a trigger.

\subsection{Missed and false triggers}

Other than the correct detections, there are missed $P$ waves and false triggers. Among three missed $P$ waves, two records were completely missed and one record missed the $P$ but was triggered by the $S$. Fig. 5 
(a) Waveform (Quiet)

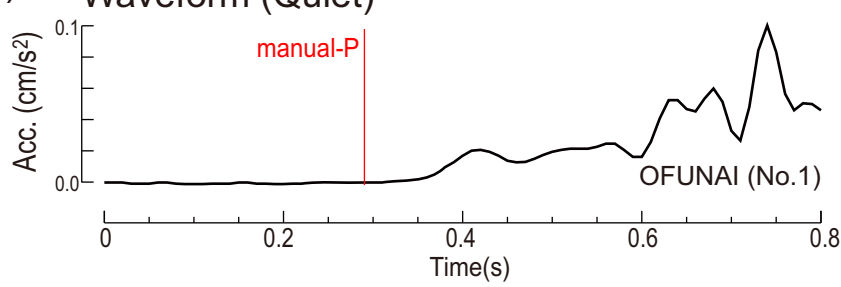

(b) Trigger $\left(\Delta \mathrm{T}^{\mathrm{pd}}>0.015\right)$

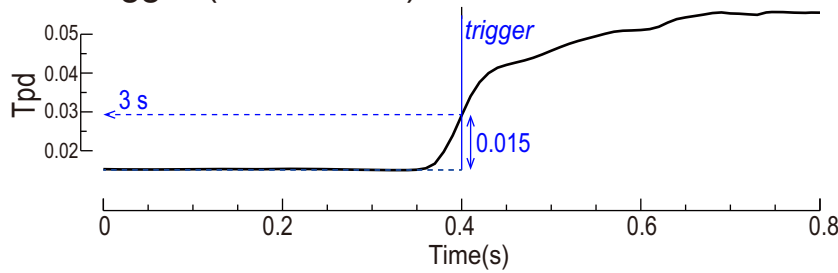

(c) Refinement Step1

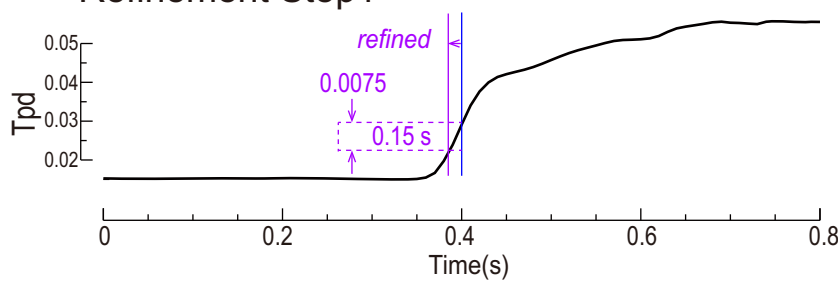

(d)

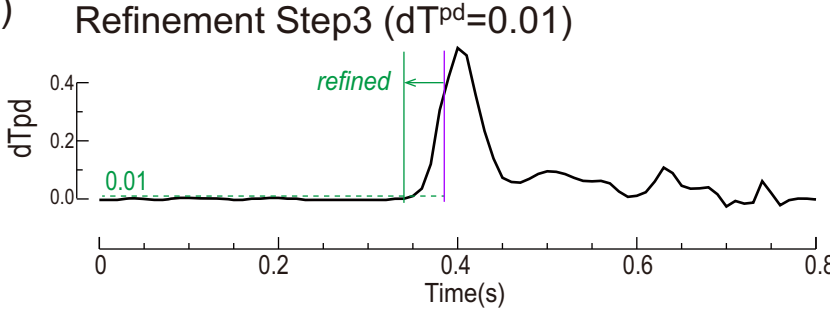

Waveform (Noisy)

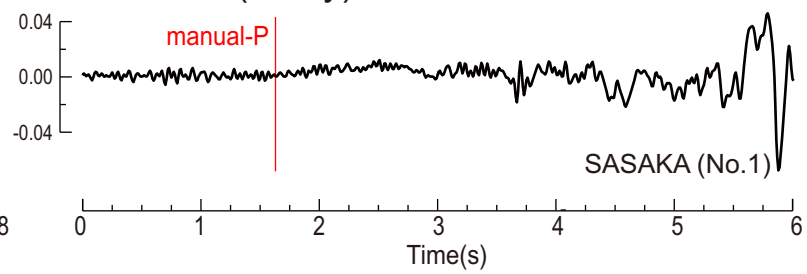

Trigger $\left(\Delta \mathrm{T}^{\mathrm{pd}}>0.015\right)$

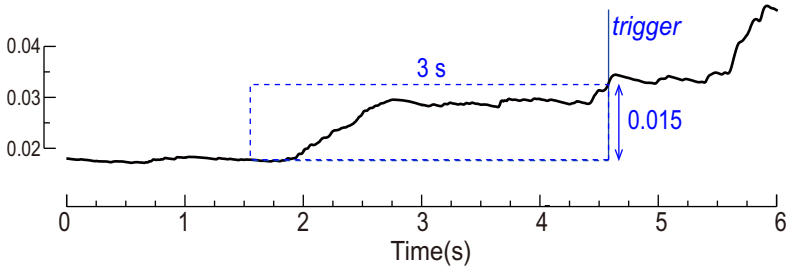

Refinement Step2

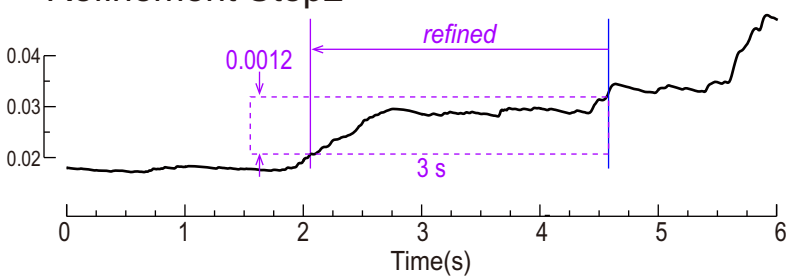

Refinement Step3 $\left(\mathrm{dT}^{\mathrm{pd}}=0.01\right)$

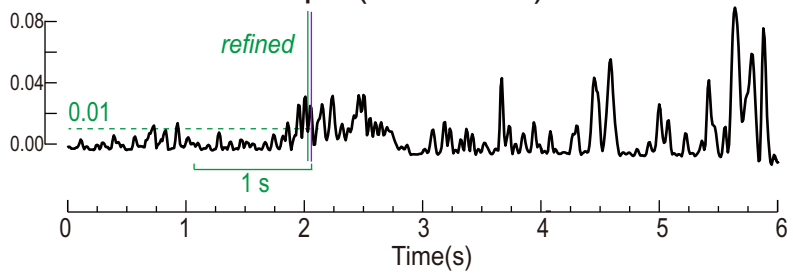

Figure 1. Examples of the trigger time refinement on the seismic records in quiet (left) and noisy (right) environments, respectively. (a) Acceleration waveforms, (b) $T^{p d}$ triggers, (c) $T^{p d}$ refinements and (d) $\mathrm{d} T^{p d}$ refinements. The left waveform is Tohoku foreshock (earthquake no.1 in Table 1 ) at station OFUNAI and the right waveform is the same event at station SASAKA.

shows the acceleration and $T^{p d}$ traces of these three records. Due to high background noise, the increase of the $T^{p d}$ was very slow, and the change of $T^{p d}$ was not large enough to exceed the threshold in $3 \mathrm{~s}$. The $P$-wave arrival at the OURI station (Fig. $5 \mathrm{c}$ ) was contaminated by a previous small earthquake, so it was extremely difficult to correctly determine the arrival time.

We had 228 extra triggers in the 606 waveforms that were not associated with the target earthquake. Using theoretical arrival times of the earthquakes in the JMA catalogue, 55 and 18 picks were identified as $P$ and $S$ waves, respectively, of small earthquakes in the catalogue. The remaining 155 picks were caused by noise or earthquakes not in the catalogue. Since an earthquake catalogue is not complete after a large earthquake, it is hard to tell whether or not these were real seismic phases. Although they may be earthquake triggers, we define the picks not associated with earthquakes in the JMA catalogue as false triggers.

\subsection{Grid search for the threshold of detection}

We tried to find a threshold of the trigger ( $c_{1}$ in eq. 6 ), which shows the best performance for detecting the large target earthquakes and does not trigger on other phases or noise. There is a clear trade-off between these criteria. We performed a grid search for the following range of parameters: $c_{1}=(0.010,0.011, \ldots, 0.020)$.

Fig. 6 shows the relationship between the number of missed $P$ waves and false triggers for the different thresholds. As expected, the number of the missed $P$ waves increases as the threshold increases and the number of false triggers increases as the threshold decreases. However, there seems to be a lowerbound for the number of missed $P$ waves. The number of the missed $P$ waves does not change significantly for $c_{1} \leq 0.016$. To minimize both the number of false triggers and missed $P$ waves for the target earthquakes, we selected $c_{1}=0.015$.

\subsection{Estimating location from the automatic triggers}

To evaluate the effect of the $P$-wave detection on the location estimation, we compared locations of earthquakes using JMA manual picks and our automatic picks. We used 12 inland earthquakes in our data set which have good azimuthal station coverage. The JMA2001 1-D velocity structure model (Ueno et al. 2002) was used with the program Fasthypo (Herrmann 1979) for calculating the locations. 


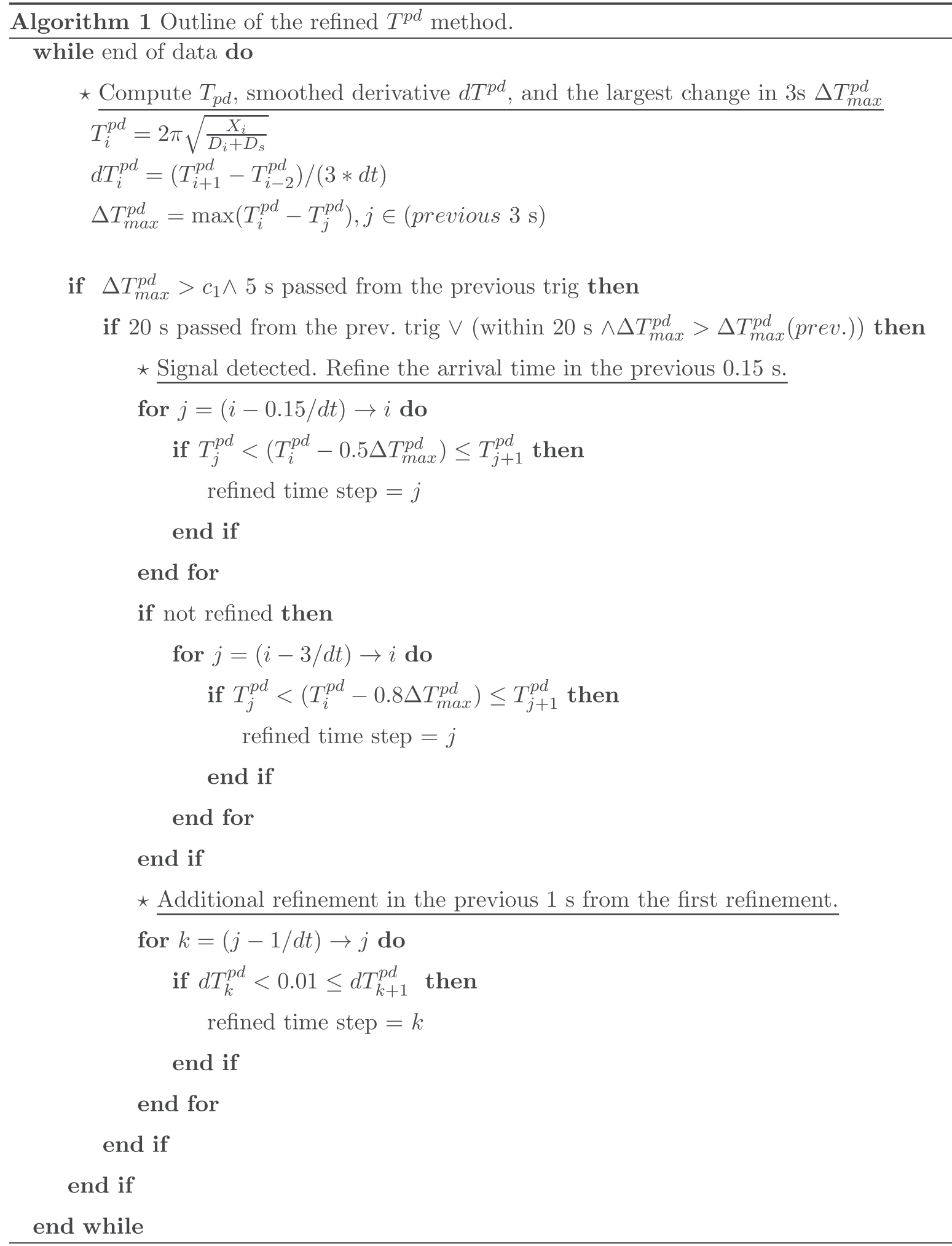

Figure 2. Pseudo-code of the refined $T^{p d}$ method.

The differences between the two epicentres are shown in Table 1 . The differences were less than $1 \mathrm{~km}$ for most of the earthquakes except the immediate aftershocks of the Fukushima-Hamadori earthquake. As we have shown in the Fig. 4, for these events, the $P$ wave arrival times were contaminated by a small earthquake right before the target earthquake. In general, the difference of the $P$-wave arrival times determined by the JMA manual processing and the automatic triggers in our method is very small. As a result, we consider that the location errors of the earthquakes using our method are small. The earthquake early warning system determines the 

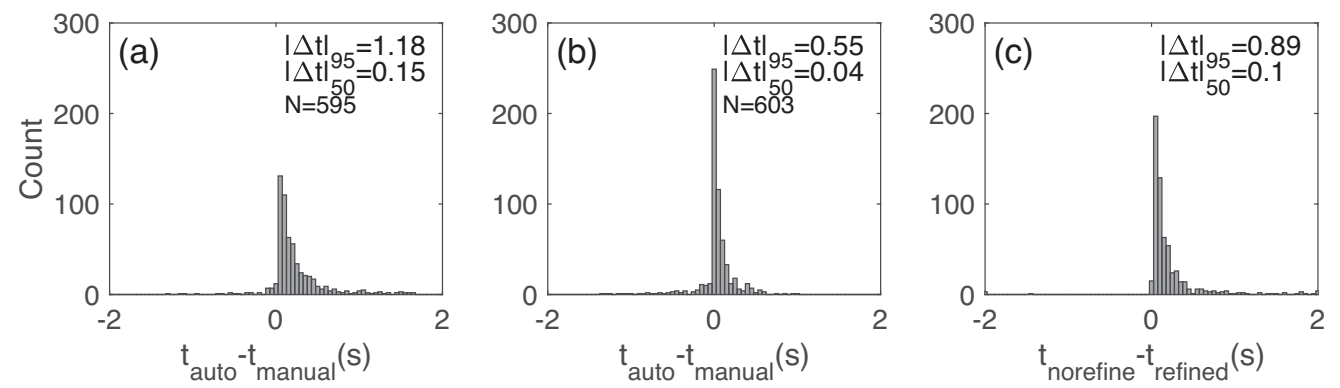

Figure 3. Effect of the refinement for the accuracy of $P$-wave arrival times. (a) No refinement, (b) with refinement and (c) the difference between (a) and (b). The number of the correct triggers, and the interval of the 95 and 50 per cent of the data are also shown.

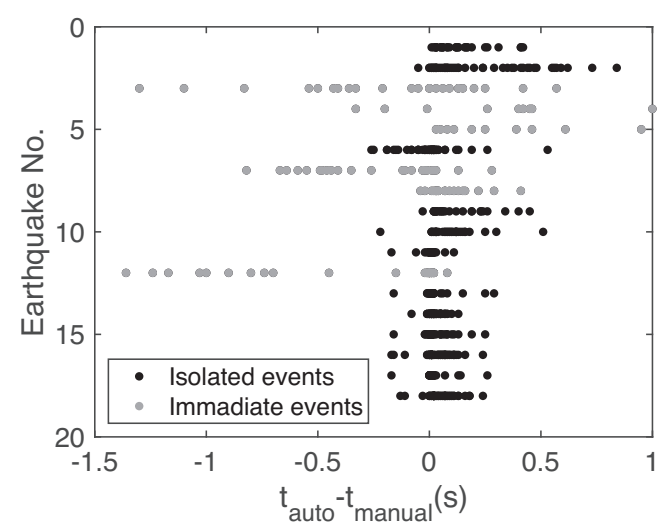

Figure 4. For each earthquake, difference between $P$-wave arrival time determined manually by JMA and automatic detection in this study. The vertical axis corresponds to the earthquake number in Table 1. The black and grey symbols indicate isolated events and immediate aftershocks, respectively.

location using a few early triggers; therefore, the accuracy of these triggers is important for obtaining a good location. In noisy environments, the refinement process proposed in this paper should provide better estimates of $P$-wave arrival times and improve the determined location when using data with high noise levels.

\section{DISCUSSION}

\subsection{Choice of parameters}

The performance of the $P$-wave detection by the $T^{p d}$ method depends on the parameter tuning. The optimal parameters may be different depending on the data set, but we propose this formulation of the method so that parameter tuning is not needed for every new data set. We tried to select parameters that were less dependent on the data set. We use two parameters for the $P$-wave detection and six parameters for the refinement. Seven of the parameters were predetermined by the trial and error, as explained in the following.

The $T^{p d}$ method uses two parameters to detect the $P$-wave arrivals (a threshold of $\Delta T_{\max }^{p d}$ and its time window). These two parameters have a trade-off: a smaller threshold of $\Delta T_{\max }^{p d}$ will be suitable for a narrow time window, and the threshold of $\Delta T_{\max }^{p d}$ becomes large if the time window increases. Therefore, we fixed the time window as $3 \mathrm{~s}$ and searched for the threshold of $\Delta T_{\max }^{p d}$. This time window was determined so that we can capture the increase of $\Delta T_{\max }^{p d}$ properly. In a noisy environment, the increase of $\Delta T_{\max }^{p d}$ is very slow, and we may miss the $P$-wave arrival if the window is too short. On the other hand, the width of the time window restricts the detection of multiple signals in a short time, so the time window cannot be too long. After trial and error, we found that the $3 \mathrm{~s}$ window was suitable to detect 99 percent $(603 / 606)$ of the $P$-wave arrivals in our data set. The best threshold with this time window was determined by the grid search (Fig. 6).

In the refinement process, we use two parameters (time window and threshold) for each refinement scheme (six parameters in total). The growth of $T^{p d}$ is different depending on the background noise level. In a quiet environment, $T^{p d}$ increases very rapidly (Fig. 1a). We see a sharp increase of $T^{p d}$ in a short time (less than $0.1 \mathrm{~s}$ ) and a monotonic function. For this type of waveform, a short time window and smaller threshold $\left(0.15 \mathrm{~s}\right.$ and 50 per cent of $\left.\Delta T_{\max }^{p d}\right)$ of the refinement are effective (eq. 7). The onset of the increase of $T^{p d}$ is clearer on the derivative of $T^{p d}$, since it changes from negative to positive at the onset. Therefore, another refinement is performed by using $\mathrm{d} T^{p d}$. This additional refinement was performed at most $1 \mathrm{~s}$ and the threshold was set to 0.01 , to capture the time of the zero-crossing (eq. 9).

In a noisy environment, $T^{p d}$ increases gradually and the onset is more difficult to determine than the quiet environment (Fig. 1b). The first refinement cannot find the onset of the waveforms and the second refinement with a longer time window and larger threshold (3 $\mathrm{s}$ and 80 percent of $\Delta T_{\max }^{p d}$ ) is applied (eq. 8). The additional refinement using $\mathrm{d} T^{p d}$ is not very effective due to the quick variation of $\mathrm{d} T^{p d}$.

With these three refinement schemes, the method can be applied to a data set with both high and low noise levels. We avoid using the direct amplitude of $T^{p d}$ as a threshold, since it may depend on the data set. Instead, we use the change of $T^{p d}$ and the derivative of $T^{p d}$ as a threshold.

\subsection{Comparison with the STA/LTA method}

The STA/LTA method is one of the most widely used methods for $P$-wave detection (e.g. Allen 1978). We compared the performance of $T^{p d}$ method to the STA/LTA method. We used $C_{3}=0.021934$ and $C_{4}=0.000227$ for the parameters of the STA and LTA, respectively (Allen 1978; Yamada et al. 2014), and performed a grid search for the following range of the STA/LTA threshold: $C_{5}=(6,7, \ldots, 30)$ (Allen 1978).

The robustness under noisy conditions depends on the threshold level of the detection. As we decrease the threshold, more signals are detected, but there are more false triggers. One way to evaluate the robustness is to examine the trade-off between missed $P$ waves of target earthquakes and false triggers. Fig. 6 shows this relationship for the STA/LTA and $T^{p d}$ methods. The results with various thresholds used in the grid search are shown in this plot. The $T^{p d}$ method 
(a)

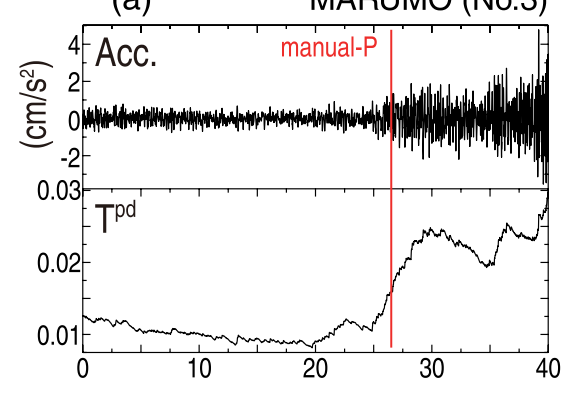

(b)

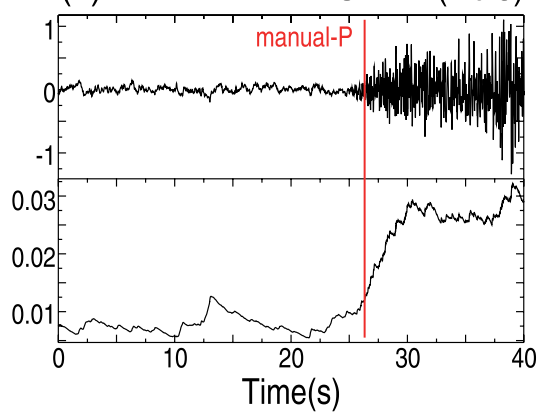

(c)

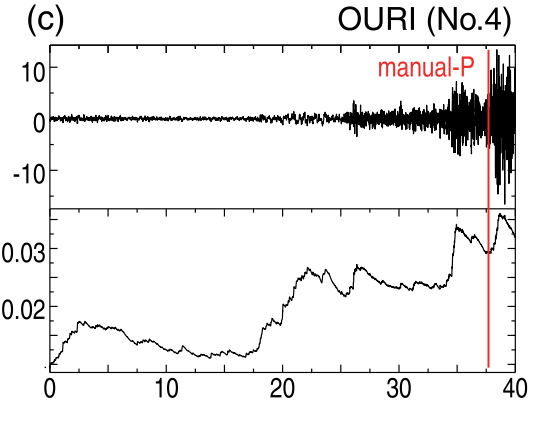

Figure 5. Acceleration and $T^{p d}$ waveforms for missed $P$-wave arrivals. (a) Earthquake no. 3 in Table 1 at station MARUMO, (b) earthquake no. 3 at station RYOKAM and (c) earthquake no. 4 at station OURI.

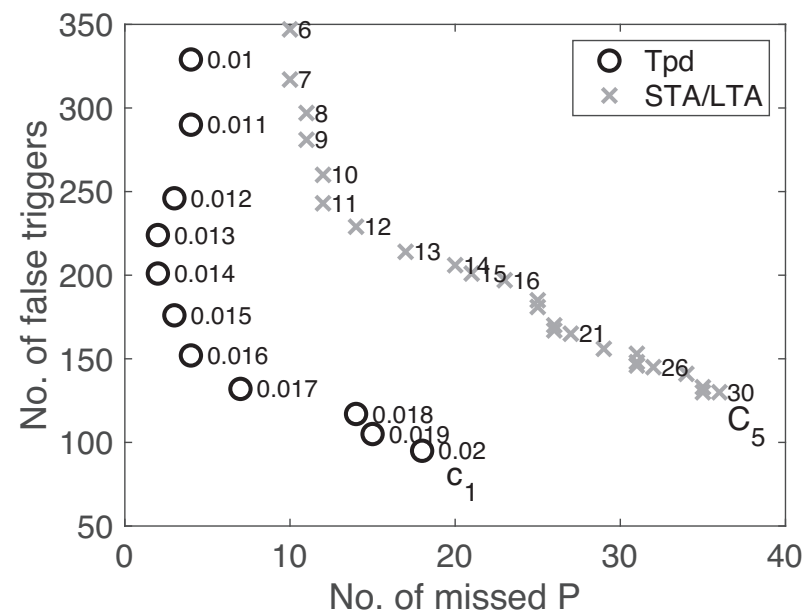

Figure 6. Relationship between the number of missed $P$ waves of target earthquakes and false triggers in our test data set for $T^{p d}$ and STA/LTA. The numbers indicate the threshold of the trigger $\left(\mathrm{c}_{1}\right.$ for $T^{p d}$ and $\mathrm{C}_{5}$ for STA/LTA).

shows better performance in detecting $P$-wave arrivals of the target earthquakes with a smaller number of false triggers, suggesting more robustness in the noisy environment.

Fig. 7 shows an example of $T^{p d}$ and STA/LTA traces of a noisy records in our test data set. Both trigger methods show a sharp change at the $P$-wave arrival, but $T^{p d}$ does not change significantly afterwards, which makes it easier to distinguish between the ambient noise and $P$-wave signal. If we use the best threshold of STA/LTA in Fig. $6\left(C_{5}=12\right)$, the STA/LTA method missed the $P$ wave and detected the $S$ wave in Fig. 7(a). As for the waveform in Fig. 7(b), the STA/LTA method detected both the $P$-wave arrival and small noise right before it, whereas the $T^{p d}$ method triggers only on the $P$-wave arrival.

\subsection{Test with continuous data of the 2016 Kumamoto sequence}

Since the method and parameter tuning were developed using the 2011 Tohoku sequence, we also tested the method on the 2016 Kumamoto sequence, without redeterming the parameters. The Kumamoto earthquakes are shallow onshore events, compared to the offshore subduction events of the Tohoku sequence. We applied the $T^{p d}$ method to the continuous seismic recordings of the Kumamoto sequence. We used seismic data recorded by Hi-net and the JMA strong motion seismic network on 2016 April 14-16. We ran the $T^{p d}$ method on the entire continuous data set to detect $P$ phases, and then extracted the phases associated with 18 large earthquakes with maximum JMA seismic intensity $\geq 5$ lower. We defined the phases as correct detections, if they were within $2 \mathrm{~s}$ of the theoretical $P$-wave arrival times, calculated using the JMA locations.

The numbers of the correct $P$-wave detections for the closest 30 stations from the epicenter are shown in Supporting Information Table S1. The detection performance is satisfactory (only one or no $P$-wave arrivals are missed) for most of the events. The exceptions are four earthquakes (nos. 6, 12, 17 and 18) where nos. 6, 12 and 17 are immediate aftershocks within 7 min after the previous large earthquakes and the $P$-wave detection rate is less than 70 per cent of the 30 stations. The $T^{p d}$ method with parameters determined from the Tohoku earthquake provides good results on the Kumamoto sequence.

We evaluate possible incorrect $P$-wave detections using the continuous data $1 \mathrm{hr}$ before the Kumamoto earthquake (20:00-21:00 on 2016 April 14). There are no earthquakes in the JMA seismic catalogue around the Kumamoto region, so this is a very quiet period. We run the $T^{p d}$ method on the 1-hr continuous data with 233 stations and detected 209 single-station triggers. Although the number seems to be large, some stations are very noisy and triggered frequently. For the Japanese earthquake early warning to the public, triggers at multiple stations are required to avoid a false alarm. Therefore, we classify a trigger as noise if it is only one trigger among the 10 closest stations. With this filter, all 209 triggers described above are classified as noise, not earthquakes. Therefore, we have no false positive detections that are classified as earthquakes. Although this filter causes the delay of the warning, it is the simplest way to avoid false alarms for a place with a dense seismic network.

\subsection{Applicability to real-time processing}

We measured the speed of computation in applying the $T^{p d}$ method on continuous waveforms. The computation time required for reading the 1-hr 997-channel data from the hard disk and applying the $T^{p d}$ method is about 36 min (operation environment: CPU, Xeon $3.46 \mathrm{GHz}$; OS, Linux CentOS 6; memory, $48 \mathrm{~GB}$; compiler, gcc ver.4.4.7). Currently, we are testing this triggering method for about 1000-channel real-time data on the same server. The program has been working continuously for several months. This program was designed to process every $1 \mathrm{~s}$ and used recursive computation. Therefore, it does not require a large memory and CPU, even when we process 1000 continuous channels. 
(a)

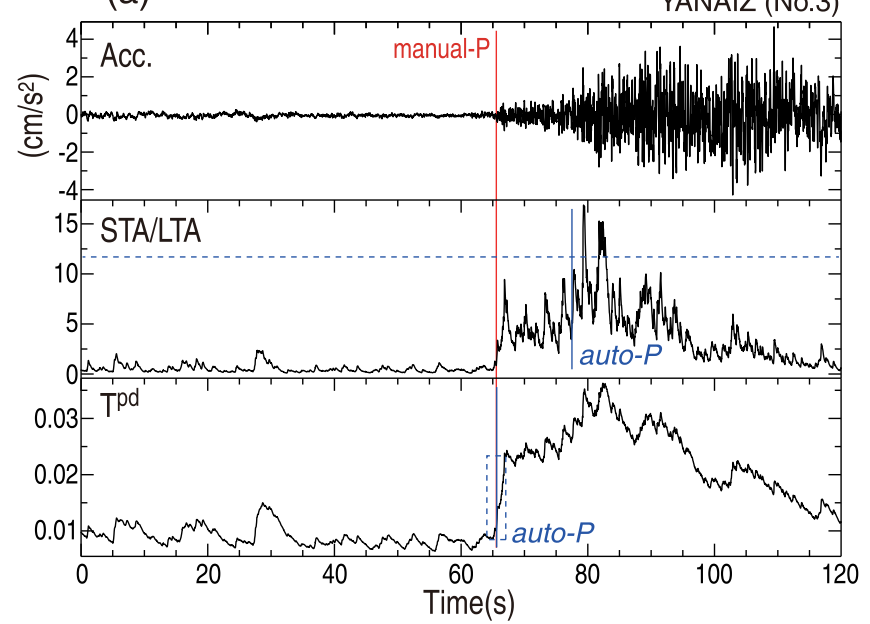

(b)

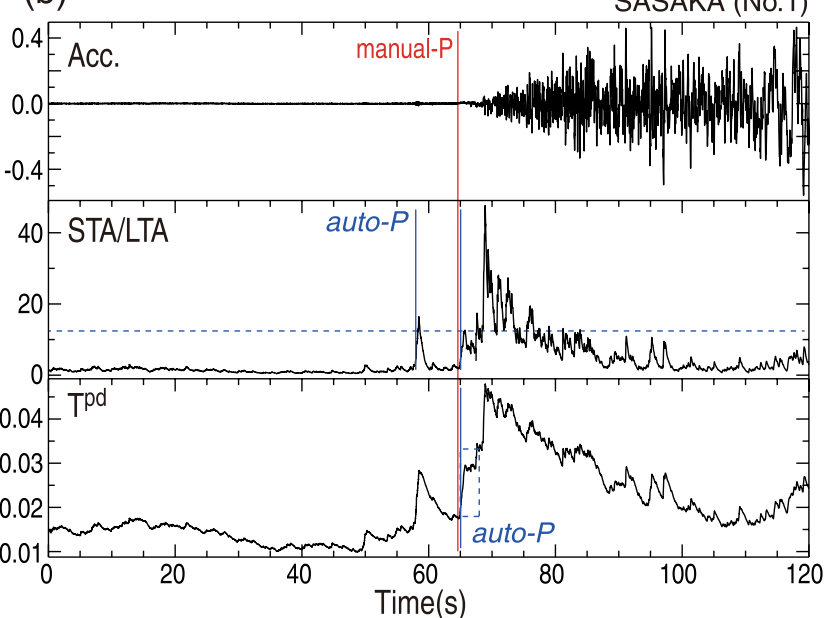

Figure 7. Example of noisy waveforms. Acceleration, STA/LTA and $T^{p d}$ waveforms from the top. JMA manual determined $P$-wave arrival (marked as manual- $P$ ) and automatic triggers using STA/LTA and refined $T^{p d}$ methods (marked as auto- $P$ ). (a) Earthquake no. 3 in Table 1 at station YANAIZ and (b) earthquake no. 1 at station SASAKA.

Note that we use the acceleration waveforms to apply the $T^{p d}$ method, but the velocity waveforms have a similar trend of $T^{p d}$, since the $T^{p d}$ is the ratio between the waveform and its derivative. We tested the velocity waveforms and found that this method was also applicable to the velocity waveforms.

\section{CONCLUSIONS}

In this paper, we tried to improve the $P$-wave detection method for real-time processing of continuous data. We used a new $T^{p d}$ methods, and proposed a refinement algorithm to determine the $P$-wave arrival time.

Applying a refinement process substantially decreases the error of the $P$-wave arrival time. For the 606 waveforms of the test data set, the median of the error was decreased from 0.15 to $0.04 \mathrm{~s}$. The number of the missed $P$ waves decreased from 11 to 3 . Our results show that the $T^{p d}$ method with the refinement has good accuracy for detecting $P$-wave arrival times for large earthquakes.

We also proposed simple detrigger and retrigger schemes, which are required for the real-time continuous system. The computation time required for processing the 1-hr 1000-channel data is about $36 \mathrm{~min}$.

Our results show that the $T^{p d}$ method has better performance for detecting $P$-wave arrivals than the STA/LTA method. It is more robust in detecting the $P$-wave arrivals of the target large earthquakes and less sensitive to false triggers. The $T^{p d}$ method is computationally fast, so it would be suitable for implementation in earthquake early warning systems.

\section{FUNDIN G}

This work was partially supported by the seismology toward research innovation with data of earthquake (STAR-E) project.

\section{ACKNOWLEDGEMENTS}

We thank the Japan Meteorological Agency (JMA) and National Research Institute for Earth Science and Disaster Resilience (NIED) for providing seismic waveform data. MY processed the data. MY and JM analysed the results and wrote the manuscript.

\section{DATA AVAILABILITY}

We use the seismic waveform data provided by the JMA and NIED. The Hi-net data provided by the NIED are available at https://do i.org/10.17598/NIED.0003 (last accessed August 2021). The JMA data were provided under resarch collaboration with the JMA. Data can be purchased from the Japan Meteorological Business Support Center.

\section{REFER E N CES}

Allen, R., 1978. Automatic earthquake recognition and timing from single traces, Bull. seism. Soc. Am., 68(5), 1521-1532.

Allen, R. \& Kanamori, H., 2003. The potential for earthquake early warning in southern California, Science, 300(5620), 786-789.

Allen, R., Gasparini, P., Kamigaichi, O. \& Böse, M., 2009. The status of earthquake early warning around the world: an introductory overview, Seismol. Res. Lett., 80(5), 682-693.

Anant, K.S. \& Dowla, F.U., 1997. Wavelet transform methods for phase identification in three-component seismograms, Bull. seism. Soc. Am., 87(6), 1598-1612.

Galiana-Merino, J.J., Rosa-Herranz, J.L. \& Parolai, S., 2008. Seismic $P$ phase picking using a kurtosis-based criterion in the stationary wavelet domain, IEEE Trans. Geosci. Remote Sens., 46(11), 3815-3826.

Hafez, A.G., Khan, M.T.A. \& Kohda, T., 2010. Clear $P$-wave arrival of weak events and automatic onset determination using wavelet filter banks, Digit. Signal Process., 20(3), 715-723.

Herrmann, R.B., 1979. Fasthypo-a hypocenter location program, Earthq. Notes, 50(2), 25-38.

Hildyard, M.W., Nippress, S.E. \& Rietbrock, A., 2008. Event detection and phase picking using a time-domain estimate of predominate period $7^{\mathrm{pd}}$, Bull. seism. Soc. Am., 98(6), 3025-3032.

Kanamori, H., 2005. Real-time seismology and earthquake damage mitigation, Annu. Rev. Earth Planet. Sci., 33, 195-214.

Kurzon, I., Vernon, F., Rosenberger, A. \& Ben-Zion, Y., 2014. Real-time automatic detectors of $P$ and $S$ waves using singular value decomposition, Bull. seism. Soc. Am., 104(4), 1696-1708. 
Leonard, M. \& Kennett, B., 1999. Multi-component autoregressive techniques for the analysis of seismograms, Phys. Earth planet. Inter., 113(14), 247-263.

Mousavi, S.M., Ellsworth, W.L., Zhu, W., Chuang, L.Y. \& Beroza, G.C., 2020. Earthquake transformer-an attentive deep-learning model for simultaneous earthquake detection and phase picking, Nat. Commun., 11(1), 3952, doi:10.1038/s41467-020-17591-w.

Nippress, S., Rietbrock, A. \& Heath, A., 2010. Optimized automatic pickers: application to the ANCORP data set, Geophys. J. Int., 181(2), 911-925.

Ross, Z.E. \& Ben-Zion, Y., 2014. Automatic picking of direct $P, S$ seismic phases and fault zone head waves, Geophys. J. Int., 199(1), 368-381.

Ross, Z.E., Meier, M. \& Hauksson, E., 2018. $P$ wave arrival picking and first-motion polarity determination with deep learning, J. geophys. Res., 123(6), 5120-5129.

Saragiotis, C.D., Hadjileontiadis, L.J. \& Panas, S.M., 2002. $\mathrm{PAI}-\mathrm{S} / \mathrm{K}:$ a robust automatic seismic $\mathrm{P}$ phase arrival identification scheme, IEEE Trans. Geosci. Remote Sens., 40(6), $1395-1404$.

Satriano, C., Lomax, A. \& Zollo, A., 2008. Real-time evolutionary earthquake location for seismic early warning, Bull. seism. Soc. Am., 98(3), 1482-1494.

Takanami, T. \& Kitagawa, G., 1988. A new efficient procedure for the estimation of onset times of seismic waves, J. Phys. Earth, 36(6), 267290.
Takanami, T. \& Kitagawa, G., 1991. Estimation of the arrival times of seismic waves by multivariate time series model, Ann. Inst. Stat. Math., 43(3), 407-433.

Ueno, H., Hatakeyama, S., Aketagawa, T., Funasaki, J. \& Hamada, N., 2002. Improvement of hypocenter determination procedures in the Japan Meteorological Agency, Q. J. Seismol., 65, 123-134.

Wessel, P. \& Smith, W., 1991. Free software helps map and display data, EOS, Trans. Am. geophys. Un., 72(441), 445-446.

Yamada, M., Tamaribuchi, K. \& Wu, S., 2014. Faster and more accurate earthquake early warning system-combination of velocity and acceleration-type seismometers, J. Japan Assoc. Earthq. Eng., 14, 2134.

Zhu, W. \& Beroza, G.C., 2019. PhaseNet: a deep-neural-network-based seismic arrival-time picking method, Geophys. J. Int., 216(1), 261-273.

\section{SUPPORTING INFORMATION}

Supplementary data are available at $G J I$ online.

Table S1. List of the large earthquakes from the 2016 Kumamoto sequence.

Please note: Oxford University Press is not responsible for the content or functionality of any supporting materials supplied by the authors. Any queries (other than missing material) should be directed to the corresponding author for the paper. 